\title{
Phytochrome A and phytochrome B regulate the biosynthesis of chlorophyll during cytokinin-dependent de-etiolation of Arabidopsis thaliana
}

\author{
Malyukova A.M. ${ }^{1,2 *}$, Doroshenko A.S. ${ }^{1}$, Danilova M.N. ${ }^{1}$ \\ ${ }^{1}$ Timiryazev Institute of Plant Physiology, RAS, Moscow, Russia \\ ${ }^{2}$ Moscow State University, Moscow, Russia \\ * email: anastasia.malukowa@gmail.com
}

Seedling greening during de-etiolation is caused by light-dependent chlorophyll biosynthesis (CB) and development of photosynthetic machinery in chloroplasts. These sweeping changes occur as a result of reprogramming of expression of the nuclear and plastid genomes. In Arabidopsis, most CB genes are encoded in the nucleus. HEMA1 HEMA3 genes encode glutamyl-tRNA reductase, that catalyzes the first step of CB. Another key enzyme in $\mathrm{CB}$ is $\mathrm{Mg}$-chelatase, which is responsible for the insertion of $\mathrm{Mg}^{2+}$ into protoporphyrin IX (CHLD, CHLI, GUN5). GUN4 stimulates CB by stabilizing the $\mathrm{Mg}$-chelatase complex in membranes. White light is the main exogenous positive regulator of $\mathrm{CB}$. Light sensing in plants is performed by photoreceptors, particularly, phytochromes (phyA-phyE) are responsible for the red light perception in A. thaliana. Phytochromes are known to positively regulate $\mathrm{CB}$. In addition to light, cytokinins have a positive effect on $\mathrm{CB}$ : they stimulate the accumulation of mRNA of CB genes, but the mechanism of this positive regulation remains unclear. Analysis of the expression of some key CB genes (HEMA1, GUN5, GUN4) by PCR after reverse transcription showed that both red and white light illumination stimulated the growth in mRNA level in wild type Landsberg erecta (Ler) and knockout mutant phyAphyB seedlings of A. thaliana. Cytokinin treatment of both Ler and phyAphyB resulted in increased level of HEMAI, GUN5, and GUN4 gene transcripts during de-etiolation under white light, but the mutant mostly had a weaker response to the hormone. During cytokinin-dependent de-etiolation under red light, the mRNA levels of some CB genes in Ler were similar to those under white light, but compared with wild type, phyAphyB had a reduced mRNA levels. Thus, phytochromes $\mathrm{A}$ and $\mathrm{B}$ mediate the positive effect of cytokinin on the expression of some CB genes during de-etiolation under both white and red light.

Acknowledgments: This research is supported by the Russian Foundation for Basic Research (grants No. 19-34-90183 and 20-04-00294A). 